EPJ Web of Conferences 70, 00002 (2014)

DOI: $10.1051 /$ epjconf/20147000002

(C) Owned by the authors, published by EDP Sciences, 2014

\title{
Higgs Boson search at the CMS experiment
}

\author{
Somnath Choudhury ${ }^{a, b}$ \\ ${ }^{1}$ CEA - Saclay, France
}

\begin{abstract}
The results of the search for the Standard Model (SM) Higgs Boson with the CMS detector in proton-proton collisions at the $\mathrm{LHC}$ at $7 \mathrm{TeV}$ center-of-mass energy are reported. A large number of the Higgs Boson decay channels in the mass range from $110 \mathrm{GeV}$ to $600 \mathrm{GeV}$ are considered, and combined upper limits on the production cross section as a function of the Higgs Boson mass are derived. The SM Higgs is excluded at $95 \%$ confidence level in the mass range between $127.5 \mathrm{GeV}$ and $600 \mathrm{GeV}$. In addition, searches for Higgs Bosons in scenarios Beyond the Standard Model (BSM) lead to improved constraints on the Higgs sector of BSM theories such as supersymmetry.
\end{abstract}

\section{Introduction}

The discovery of the mechanism of electro-weak symmetry breaking has been one of the primary goals of the Large Hadron Collider (LHC) at CERN. In the Standard Model (SM), electro-weak symmetry breaking [1-4] is achieved by the introduction of a complex scalar doublet leading to 4 physical degrees of freedom of which are the 3 "eaten" Goldstone bosons, namely the longitudinal states of the $\mathrm{W}^{+}, \mathrm{W}^{-}$and the $\mathrm{Z}$ boson, and the remaining one is a real physical scalar particle which is the Higgs boson. There are four main mechanisms for Higgs boson production in pp collisions at $\sqrt{s}$ $=7 \mathrm{TeV}$. The gluon-gluon fusion mechanism has the largest cross-section, followed in turn by vector boson fusion (VBF), associated $\mathrm{WH}$ and $\mathrm{ZH}$ production, and production in association with top quarks, $\mathrm{ttH}$. The total cross-section for the Higgs boson production mechanisms varies from 20 to 0.3 $\mathrm{pb}$ as a function of the Higgs boson mass, over the explored range. The relevant decay modes of the SM Higgs boson depend strongly on the decay branching fraction with its mass $\mathrm{m}_{\mathrm{H}}$. The results presented here are based on the following five decay modes: $\mathrm{H} \rightarrow \gamma \gamma, \mathrm{H} \rightarrow \tau \tau, \mathrm{H} \rightarrow b \bar{b}, \mathrm{H} \rightarrow \mathrm{WW}$, followed by $\mathrm{WW} \rightarrow(\ell v)(\ell v)$ decays, and $\mathrm{H} \rightarrow \mathrm{ZZ}$, followed by ZZ decays to $4 \ell, 2 \ell 2 v, 2 \ell 2 q$ and $2 \ell 2 \tau$, where $\ell$ stands for electrons or muons and $q$ for quarks.

The Higgs boson suffers from quadratically divergent self-energy corrections at high energies. Numerous extensions to the SM have been proposed to address these divergences one of which is supersymmetry, a symmetry between fundamental bosons and fermions, which results in cancellation of the divergences. The Minimal Supersymmetric extension of the Standard Model (MSSM), requires the introduction of 2 Higgs doublets leads to 5 physical Higgs bosons, $\mathrm{h}$ and $\mathrm{H}$ are the $\mathrm{CP}$-even scalar ones, $\mathrm{A}$ is the $\mathrm{CP}$-odd pseudoscalar boson and $\mathrm{H}^{+}$and $\mathrm{H}^{-}$are the charged ones. A search for MSSM Neutral and Charged Higgs Bosons in decays to tau leptons have been presented here.

\footnotetext{
${ }^{a}$ for the CMS collaboration

be-mail: somnath.choudhury@gmail.com
}

This is an Open Access article distributed under the terms of the Creative Commons Attribution License 2.0, which permits unrestricted use, distribution, and reproduction in any medium, provided the original work is properly cited. 
The SM Higgs boson search presented here uses $7 \mathrm{TeV}$ proton-proton collision data collected by the CMS detector [5] at the LHC corresponding to an integrated luminosity ranging from 4.6 to $4.8 \mathrm{fb}^{-1}$ depending on the search channel. The search for MSSM neutral and charged Higgs bosons is based on $4.6 \mathrm{fb}^{-1}$ and up to $2.3 \mathrm{fb}^{-1}$ of $7 \mathrm{TeV}$ pp collision data respectively. The CMS detector consists of a barrel assembly and two end-caps, comprising, in successive layers outwards from the collision region, the silicon pixel and strip tracker, the lead tungstate crystal electromagnetic calorimeter, the brass/scintillator hadron calorimeter, the superconducting solenoid, and gas-ionization chambers embedded in the steel return yoke for the detection of muons.

\section{Standard Model Higgs}

For a given Higgs boson mass hypothesis, the search sensitivity depends on the Higgs boson production cross section and decay branching fraction into the chosen final state, the signal selection efficiency, the Higgs boson mass resolution, and the level of standard model backgrounds with the same or a similar final state. In the low-mass range, the $b \bar{b}$ and $\tau \tau$ decay modes suffer from large backgrounds, which reduces the search sensitivity in these channels. For a low mass Higgs boson around $120 \mathrm{GeV}$, the best sensitivity is achieved in the $\gamma \gamma$ decay mode, which has a very small branching fraction, but manageable background. In the mass range $120-200 \mathrm{GeV}$, the best sensitivity is achieved in the $\mathrm{H} \rightarrow \mathrm{WW}$ channel. At higher masses, the $\mathrm{H} \rightarrow \mathrm{ZZ}$ branching fraction is large and the searches for $\mathrm{H} \rightarrow \mathrm{ZZ} \rightarrow 4 \ell$ and $\mathrm{H} \rightarrow \mathrm{ZZ} \rightarrow 2 \ell 2 v$ provide the best sensitivity. Among all decay modes, the $\mathrm{H} \rightarrow \gamma \gamma$ and $\mathrm{H} \rightarrow \mathrm{ZZ} \rightarrow 4 \ell$ channels play a special role as they provide an excellent mass resolution for the reconstructed diphoton and four-lepton final states, respectively. A search for the Standard Model Higgs boson is performed in the decay channels $\mathrm{H} \rightarrow \gamma \gamma$ [6, 7], $\mathrm{H} \rightarrow \tau \tau$ [9], $\mathrm{H} \rightarrow b \bar{b}$ [11], $\mathrm{H} \rightarrow \mathrm{WW}$ [14] and $\mathrm{H} \rightarrow \mathrm{ZZ}[12,13]$ covering the mass range of 110 to $600 \mathrm{GeV}$. A combined result [18] of all the search channels considered using the CLs method [15-17] has also been presented.

\section{$2.1 \mathrm{H} \rightarrow \gamma \gamma$}

The Higgs boson branching ratio for the decay into two photons is approximately $2 \times 10^{-3}$ between 110 and $150 \mathrm{GeV}$.The diphoton mass resolution is excellent, between 1 and $2 \%$ and the signature in this channel is two high $\mathrm{E}_{\mathrm{T}}$ isolated photons. In case of the VBF process there are two additional high $\mathrm{p}_{\mathrm{T}}$ jets that provide a further handle to discriminate the signal from the background. A signal in this channel would appear as a small and narrow peak above a large and smooth prompt di-photon background. A search is performed for a Higgs boson decaying into two photons. The analysis is done using a dataset recorded by the CMS experiment at the LHC from pp collisions at a centre-ofmass energy of $7 \mathrm{TeV}$, which corresponds to an integrated luminosity of $4.8 \mathrm{fb}^{-1}$. To improve the sensitivity of the search, selected diphoton events are subdivided into classes according to indicators of mass resolution and signal-to-background ratio. Five mutually exclusive event classes are defined as shown in Figure 1: four in terms of the pseudorapidity and the shower shapes of the photons, and a fifth class into which are put all events containing a pair of jets passing selection requirements which are designed to select Higgs bosons produced by the vector boson fusion process. Two photon classifiers are used: the minimum value of a variable $\mathrm{R}_{9}$ of the two photons, $\mathrm{R}_{9}^{\mathrm{min}}$, and the maximum pseudorapidity (absolute value) of the two photons, giving four classes based on photon properties. The variable $R_{9}$ is defined as the energy sum of $3 \times 3$ crystals centred on the most energetic crystal in the supercluster divided by the energy of the supercluster. Its value is used in the analysis to identify photons undergoing conversion. The class boundary values for $\mathrm{R}_{9}$ and pseudorapidity are the same as those used to categorize photon candidates for the photon identification cuts. These photon classifiers 


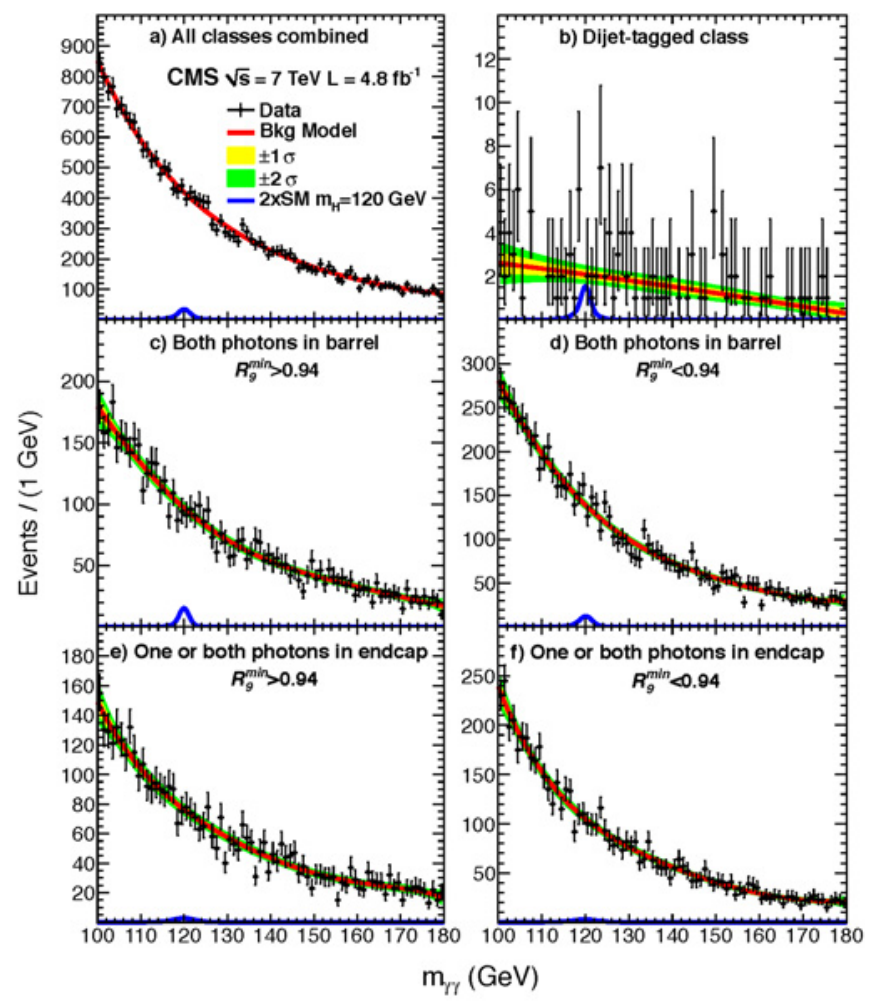

Figure 1. Background model fit to the $\mathrm{m}_{\gamma \gamma}$ distribution for the five event classes, together with a simulated signal $\left(m_{\mathrm{H}}=120 \mathrm{GeV}\right)$. The magnitude of the simulated signal is what would be expected if its cross section were twice the SM expectation. The sum of the event classes together with the sum of the five fits is also shown.

are effective in separating diphotons whose mass is reconstructed with good resolution from those whose mass is less well measured and in separating events for which the signal-to-background probability is higher from those for which it is lower. A further class of events includes any event passing a dijet tag defined to select Higgs bosons produced by the VBF process. Events in which a Higgs boson is produced by VBF have two forward jets, originating from the two scattered quarks. Higgs bosons produced by this mechanism have a harder transverse momentum spectrum than those produced by the gluon-gluon fusion process or the photon pairs produced by the background processes. By using a dijet tag it is possible to define a small class of events which have an expected signal-to-background ratio more than an order of magnitude greater than events in the four inclusive classes defined by photon properties. The additional classification of events into a dijet-tagged class improves the sensitivity of the analysis by about $10 \%$.

The local p-value quantifies the probability for the background to produce a fluctuation at least as large as observed, and assumes that the relative signal strength between the event classes follows the Monte Carlo (MC) signal model for the standard model Higgs boson. The local p-value for the cutbased approach [6] corresponding to the largest upwards fluctuation of the observed limit, at $124 \mathrm{GeV}$, has been computed to be $9.2 \times 10^{-4}(3.1 \sigma)$ in the asymptotic approximation, and $1.5 \times 10^{-3}(3.0 \sigma)$ 


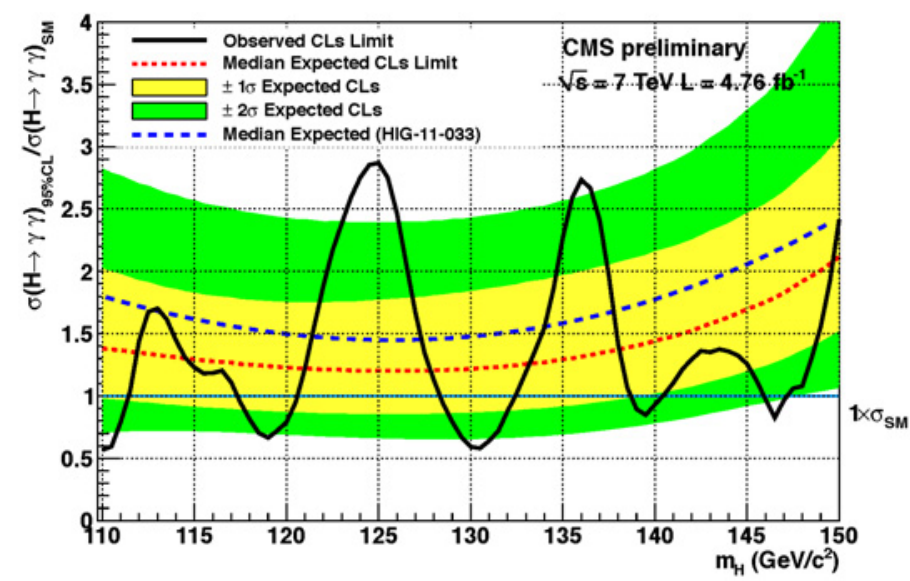

Figure 2. Background model fit to the $\mathrm{m}_{\gamma \gamma}$ distribution for the five event classes, together with a simulated signal $\left(m_{\mathrm{H}}=120 \mathrm{GeV}\right)$. The magnitude of the simulated signal is what would be expected if its cross section were twice the SM expectation. The sum of the event classes together with the sum of the five fits is also shown.

when the calculation uses pseudo-data. The combined best fit signal strength, for a SM Higgs boson mass hypothesis of $124 \mathrm{GeV}$, is $2.1 \pm 0.6$ times the SM Higgs boson cross section. Since a fluctuation of the background could occur at any point in the mass range, there is a look-elsewhere effect. When this is taken into account the probability, under the background only hypothesis, of observing a similar or larger excess in the full analysis mass range $\left(110<\mathrm{m}_{\mathrm{H}}<150 \mathrm{GeV}\right)$ is $3.9 \times 10^{-2}$, corresponding to a global significance of $1.8 \sigma$. Limits are set on the cross section of the standard model Higgs boson decaying to two photons. The expected exclusion limit at $95 \%$ confidence level is between 1.4 and 2.4 times the standard model cross section in the mass range between 110 and $150 \mathrm{GeV}$. The analysis of the data excludes, at $95 \%$ confidence level, the standard model Higgs boson decaying into two photons in the mass range 128 to $132 \mathrm{GeV}$. The largest excess of events above the expected standard model background is observed for a Higgs boson mass hypothesis of $124 \mathrm{GeV}$ with a local significance of $3.1 \sigma$ for the cut-based analysis. The global significance of observing an excess with a local significance greater than $3.1 \sigma$ anywhere in the search range $110-150 \mathrm{GeV}$ is estimated to be $1.8 \sigma$.

CMS has employed multivariate analysis for this search [7] as a development over the cut-based analysis. The search using multivariate techniques improve the search sensitivity. The di-photon multivariate analysis is trained to distinguish $\mathrm{H} \rightarrow \gamma \gamma$ events from background and identify good resolution signal events weighting them by estimated mass resolution. The expected limit is improved $20 \%$ in terms of cross section. To improve the sensitivity of the search, selected diphoton events are subdivided into classes according to the output value of a diphoton Boosted Decision Tree (BDT) which is designed to have the following properties:

1) it classifies events with signal-like kinematic characteristics with a high score,

2) it classifies good diphoton mass resolution events with a high score,

3) it classifies events with a high score from the photon identification BDT with a high score,

4) it should be mass independent, it should not select events according to the invariant mass.

Five mutually exclusive event classes are defined, four defined by the diphoton BDT output based on the signal to background ratio and mass resolution, and a fifth class into which are put all events 
containing a pair of jets passing selection requirements which are designed to select Higgs bosons produced by the vector boson fusion process. The background model is obtained by fitting polynomials of order between 3 and 5 to the observed diphoton mass distributions in each of the five event classes.

Limits are set on the cross section of a standard model Higgs boson decaying to two photons as shown in Figure 2. The expected exclusion limit at 95\% confidence level is between 1.2 and 2.1 times the standard model cross section in the mass range $110-150 \mathrm{GeV}$. The observed limit excludes at $95 \%$ confidence level a standard model Higgs boson decaying into two photons in the mass ranges 110.0111.0, 117.5-120.5, 128.5-132.5, 139.0-140.5 and 146.0-147.5 GeV. The largest excess of events over the expected background is observed around $125 \mathrm{GeV}$. Taking into account the look-elsewhere effect in the search range $110-150 \mathrm{GeV}$ the excess has a global significance of 1.6 standard deviations. More data is required to ascertain the origin of this excess at $125 \mathrm{GeV}$.

\section{$2.2 \mathrm{H} \rightarrow \tau \tau$}

The $\mathrm{H} \rightarrow \tau \tau$ search [9] is performed using the final-state signatures $\mathrm{e} \mu$, e $\tau_{h}$ and $\mu \tau_{h}$, where electrons and muons arise from leptonic $\tau$-decays and $\tau_{h}$ denotes hadronic tau lepton decays. Each of these three categories is further divided into three exclusive sub-categories according to the nature of the associated jets in the event. The gluon-fusion production mechanism has the largest production cross section. However, in the mass region of interest, background from Drell-Yan production of tau pairs overwhelms the expected Higgs boson signal. This search therefore relies upon the signature of Higgs bosons produced via vector boson fusion $(\mathrm{VBF})$ or in association with a high- $p_{\mathrm{T}}$ jet. In the former case, the distinct topology of two jets with a large rapidity separation greatly reduces the background. In the latter, requiring a high- $p_{\mathrm{T}}$ jet both suppresses background, and improves the measurement of the tau-pair invariant mass. The search has been categorized into three groups:

- VBF category: At least two jets with $p_{\mathrm{T}}>30 \mathrm{GeV},\left|\Delta \eta_{j j}\right|>4.0, \eta_{1} \cdot \eta_{2}<0$ and a dijet invariant mass $m_{\mathrm{jj}}>400 \mathrm{GeV}$, with no other jet with $p_{\mathrm{T}}>30 \mathrm{GeV}$ in the rapidity region between the two jets.

-Boosted category: One jet with $p_{\mathrm{T}}>150 \mathrm{GeV}$, and, in the e $\mu$ channel, no b-tagged jet with $p_{\mathrm{T}}>20$ $\mathrm{GeV}$.

-0/1 Jet category: No more than one jet with $p_{\mathrm{T}}>30 \mathrm{GeV}$, and if such a jet is present, it must have $p_{\mathrm{T}}<150 \mathrm{GeV}$.

To distinguish the Higgs boson signal from the background, the tau-pair mass is reconstructed using a maximum likelihood technique. The algorithm estimates the original momentum components of the two taus by maximizing a likelihood with respect to free parameters corresponding to the missing neutrino momenta, subject to kinematic constraints. Other terms in the likelihood take into account the tau-decay phase space and the probability density in the tau transverse momentum, parametrized as a function of the tau-pair mass. This algorithm yields a tau-pair mass with a mean consistent with the true value, and distribution with a nearly Gaussian shape. The standard deviation of the mass resolution is estimated to be $21 \%$ at a Higgs boson mass of $130 \mathrm{GeV}$, compared with $24 \%$ for the (non-Gaussian) distribution of the invariant mass spectrum reconstructed from the visible tau-decay products in the inclusive selection. The resolution improves to $15 \%$ in the boosted and VBF categories where the Higgs boson is produced with significant transverse momentum.

In each of these nine categories a search is performed for a broad excess in the reconstructed di-tau invariant mass distribution. The largest source of irreducible background is $\mathrm{Z} \rightarrow \tau \tau$ which is estimated using an observed sample of $\mathrm{Z} \rightarrow \mu \mu$ events, where the reconstructed muons are replaced by the reconstructed particles from simulated tau decays, a procedure called "embedding". The normalization for this process is determined from the measurement of the CMS measured cross section [10]. The reducible backgrounds $(\mathrm{W}+$ jets, multijet production, $\mathrm{Z} \rightarrow \mathrm{ee}$ ) are also evaluated from control samples 


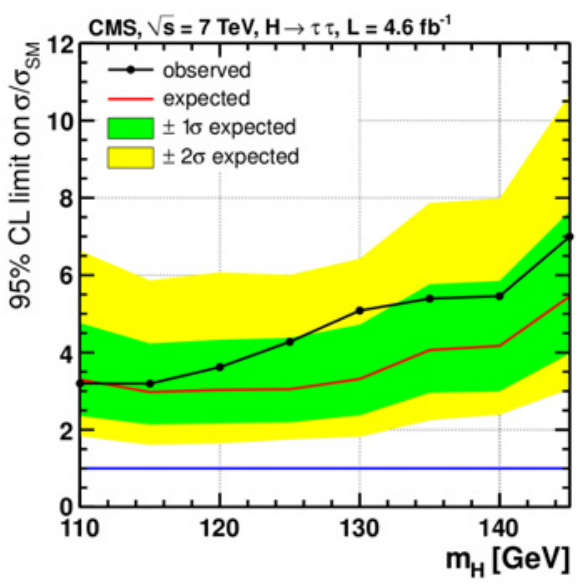

Figure 3. The expected one- and two-standard-deviation ranges and observed $95 \%$ CL upper limits on cross section normalized to the SM expectation as a function of the Higgs boson mass $\mathrm{m}_{\mathrm{H}}$.

in data. The QCD multijet background is evaluated using the ratio of opposite-sign (OS) to same-sign (SS) di-tau events and relaxed lepton isolation after an estimate of the $\mathrm{W}+$ jets background using the high transverse mass $\left(\mathrm{m}_{\mathrm{T}}{ }^{\ell E_{\mathrm{T}}^{\text {miss }}}\right)$ side-band $\mathrm{W}$ boson enriched region and extrapolating it to the signal region. The $\mathrm{Z}+$ jets background is evaluated from fake rate and OS/SS ratio with the shape from simulation. The top pair produced events and diboson contribution is estimated using simulation.

No excess of events is observed in the tau-pair invariant-mass spectrum. For a standard model Higgs boson in the mass range of 110-145 GeV upper limits at $95 \%$ confidence level (CL) on the production cross section are determined as seen in Figure 3. We exclude a Higgs boson with mass $\mathrm{m}_{\mathrm{H}}$ $=115 \mathrm{GeV}$ with a production cross section 3.2 times of that predicted by the standard model.

\section{$2.3 \mathrm{H} \rightarrow b \bar{b}$}

At the LHC the main SM Higgs boson production mechanism is gluon fusion, with a cross section of $17 \mathrm{pb}$ for Higgs boson mass $\mathrm{m}_{\mathrm{H}}=120 \mathrm{GeV}$. However, in this production mode, the detection of the $\mathrm{H}$ to $b \bar{b}$ decay is considered nearly impossible due to overwhelming dijet production expected from quantum-chromodynamic (QCD) interactions. The same holds true for the next most copious production mode, through vector-boson fusion, with a cross section of $1.3 \mathrm{pb}$. Processes in which a low-mass Higgs boson is produced in association with a vector boson have cross sections of 0.66 $\mathrm{pb}$ and $0.36 \mathrm{pb}$ for $\mathrm{WH}$ and $\mathrm{ZH}$, respectively. A search for the standard model Higgs boson $(\mathrm{H})$ decaying to a pair of bottom quarks [11] when produced in association with weak vector bosons (V) is reported for the following modes: $\mathrm{W}(\mu \nu) \mathrm{H}, \mathrm{W}(\mathrm{e} v) \mathrm{H}, \mathrm{Z}(\mu \mu) \mathrm{H}, \mathrm{Z}(\mathrm{ee}) \mathrm{H}$ and $\mathrm{Z}(v v) \mathrm{H}$. The search is performed in a data sample corresponding to an integrated luminosity of $4.7 \mathrm{fb}^{-1}$, recorded by the CMS detector in proton-proton collisions at the LHC with a center-of-mass energy of $7 \mathrm{TeV}$. The background processes to VH production are vector-boson + jets, $t \bar{t}$, single-top, dibosons (VV) and QCD multijet production. The event selection for the BDT analysis is based first on the kinematic reconstruction of the vector bosons and the Higgs boson decay into two b-tagged jets. Backgrounds are then substantially reduced by requiring a significant boost in the $p_{\mathrm{T}}$ of the vector boson and the Higgs boson. The boost requirements in the $\mathrm{Z}(\ell \ell) \mathrm{H}$ and $\mathrm{WH}$ analyses are $p_{\mathrm{T}}>100 \mathrm{GeV}$ and $p_{\mathrm{T}}>150$ $\mathrm{GeV}$, respectively. The corresponding efficiencies for signal events are approximately $25 \%$ and $10 \%$. 


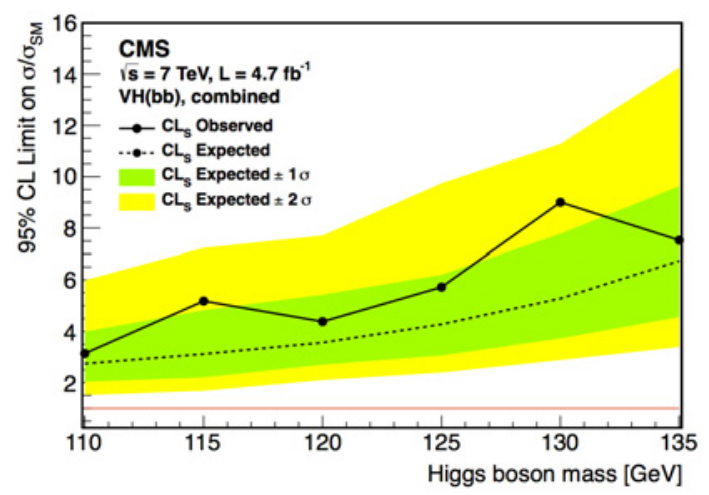

Figure 4. Expected and observed 95\% CL upper limits on the product of the VH production cross section times the $\mathrm{H}$ to bb branching ratio, with respect to the expectations for a standard model Higgs boson, for the BDT analysis. Shown are the median expected limit (dashed), one- (green) and two-sigma (yellow) bands, and the observed limits (solid with points).

For the $\mathrm{Z}(v v) \mathrm{H}$ analysis the boost requirement is $p_{\mathrm{T}}>160 \mathrm{GeV}$. Appropriate control regions that are orthogonal to the signal region are identified in data and used to adjust the Monte Carlo simulation normalization for the most important background processes: $\mathrm{W}+$ jets and $\mathrm{Z}+$ jets (with light- and heavy-flavor jets), and top production.

No significant excess of events above the expectation from background is observed. Upper limits on the $\mathrm{VH}$ production cross section times the $\mathrm{H}$ to $b \bar{b}$ branching ratio, with respect to the expectations for a standard model Higgs boson, are derived for a Higgs boson in the mass range 110 to $135 \mathrm{GeV}$ and is shown in Fig. 4. In this range, the observed 95\% confidence level upper limits vary from 3.4 to 7.5 times the standard model prediction; the corresponding expected limits vary from 2.7 to 6.7 times the standard model prediction. The limits are based on the observed event count and background estimate in signal-enriched regions defined by the output discriminant of a boosted-decision-tree algorithm (BDT analysis). As a cross-check, limits are also derived from the observed event count in the invariant mass distribution of $\mathrm{H} \rightarrow b \bar{b}$ candidates ( $\mathrm{m}(\mathrm{jj})$ analysis).

\section{$2.4 \quad H \rightarrow Z Z$}

The $\mathrm{H} \rightarrow \mathrm{ZZ} \rightarrow 4 \ell$ channel [12] is the cleanest channel and it is often referred to as the "golden channel". The signal consists of four isolated leptons providing a very clean signature, characterized by four isolated leptons with appropriate flavour and charge combinations, so that one of the two pairs can be required not to be on-shell. The Higgs boson branching ratio for this channel is rather small, approximately one per mille at high mass and even lower for masses below $2 \times \mathrm{m}_{\mathrm{W}}$ but the background is very small, consisting mainly of irreducible continuum $\mathrm{ZZ}$ production and to a lesser extent, $\mathrm{Z}+$ jets and especially $\mathrm{Z} b \bar{b}$. The four-lepton invariant mass resolution is excellent and ranges between 1 and $2 \%$. The $p_{\mathrm{T}}$ of the lower $p_{\mathrm{T}}$ leptons is rather small and one of the most important features of the analysis is the achievement of a very high lepton efficiency down to very low $p_{\mathrm{T}}$. A search for a Higgs boson in the four-lepton decay channel $\mathrm{H} \rightarrow \mathrm{ZZ}$, with each $\mathrm{Z}$ boson decaying to an electron or muon pair, is reported. The search covers Higgs boson mass hypotheses in the range $110<\mathrm{m}_{\mathrm{H}}<$ $600 \mathrm{GeV}$. The analysis uses data corresponding to an integrated luminosity of $4.7 \mathrm{fb}^{-1}$ recorded by the CMS detector in pp collisions at $\sqrt{s}=7 \mathrm{TeV}$ from the LHC. Seventy-two events are observed 

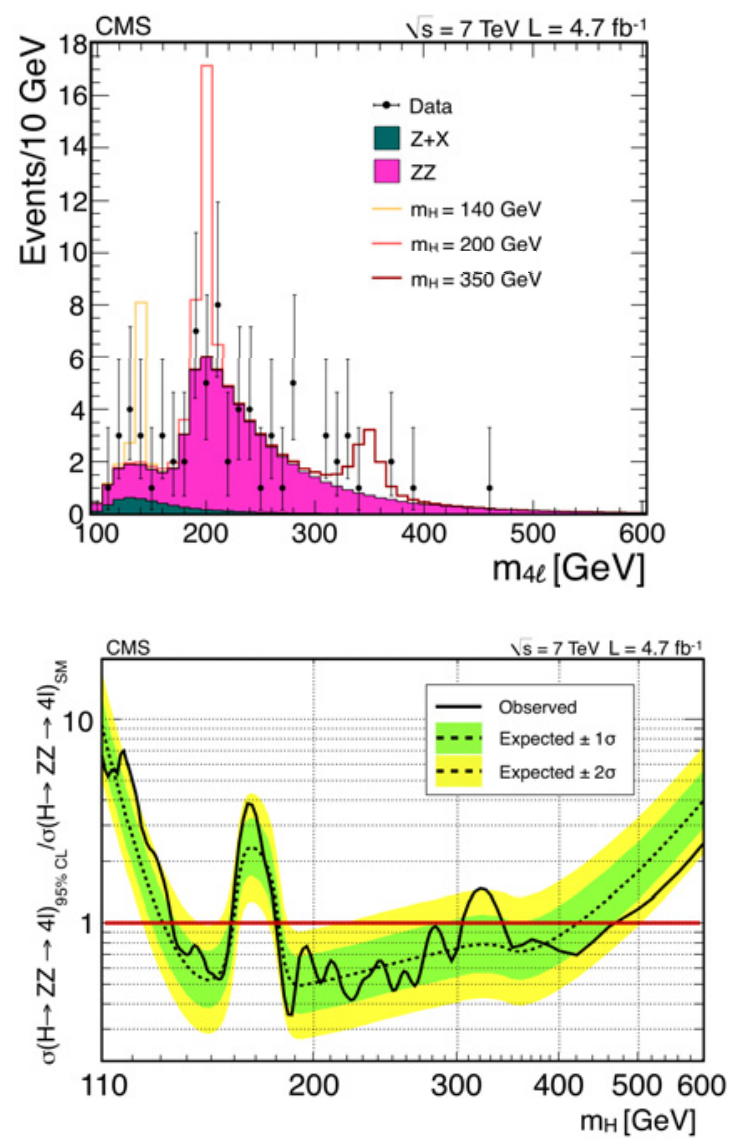

Figure 5. (Left) Distribution of the four-lepton reconstructed mass for the baseline selection in the sum of the $4 l$ channels. Points represent the data, shaded histograms represent the signal and background expectations. The samples correspond to an integrated luminosity of $\mathrm{L}=4.7 \mathrm{fb}^{-1}$. (Right) The mean expected and the observed upper limits at $95 \% \mathrm{CL}$ on $\sigma(\mathrm{pp} \rightarrow \mathrm{H}+\mathrm{X}) \times \mathrm{BR}(\mathrm{ZZ} \rightarrow 4 \ell)$ for a Higgs boson in the mass range $110-600 \mathrm{GeV}$, the limits are calculated relative to their expected SM Higgs boson prediction, for an integrated luminosity of 4.7 $\mathrm{fb}^{-1}$ using the CLs approach. The expected ratios for the SM is presented. The results are obtained using a shape analysis method.

with four-lepton invariant mass $\mathrm{m}_{4 \ell}>100 \mathrm{GeV}$ (with thirteen below $160 \mathrm{GeV}$ ), while $67.1 \pm 6.0(9.5$ \pm 1.3 ) events are expected from background. The four-lepton mass distribution is consistent with the expectation of standard model background production of ZZ pairs. Upper limits at $95 \%$ confidence level exclude the standard model Higgs boson in the ranges $134-158 \mathrm{GeV}, 180-305 \mathrm{GeV}$, and 340-465 $\mathrm{GeV}$. Small excesses of events are observed around masses of $119.5,126$, and $320 \mathrm{GeV}$ as seen in Figure 5, making the observed limits weaker than expected in the absence of a signal. The largest excess is observed at $119.5 \mathrm{GeV}$, that corresponds to a local significance of $2.5 \mathrm{\sigma}$.

A search for the Standard Model Higgs boson in the $\mathrm{H} \rightarrow \mathrm{ZZ} \rightarrow 2 \ell 2 v$ decay channel [13], where $\ell$ $=\mathrm{e}$ or $\mu$, in pp collisions at $\sqrt{s}=7 \mathrm{TeV}$ with $4.6 \mathrm{fb}^{-1}$ of data, has been performed to explore the high mass search range. The final state of this analysis is identified as a same-flavour, opposite-charge high- $p_{\mathrm{T}}$ lepton pair consistent with the $\mathrm{Z}$ boson mass, and large missing energy due to neutrinos. The $\mathrm{WW}$ and $\mathrm{ZZ}$ backgrounds that contaminate the signal region are estimated from control regions with opposite-flavour lepton pairs, while the $\mathrm{Z}+$ jets one, which depends on the missing energy reconstruction in the detector, is modeled by kinematically reweighting a $\gamma+$ jets sample. No significant excess has been observed above the background expectation, and upper limits have been set on the Higgs boson production cross section. The presence of the standard model Higgs boson in the high mass range of $270-440 \mathrm{GeV}$ has been excluded at $95 \%$ confidence level. 


\section{$2.5 \mathrm{H} \rightarrow \mathrm{WW}$}

A search for a Higgs boson decaying to a pair of $\mathrm{W}$ bosons in the CMS detector has been presented. The $\mathrm{H} \rightarrow \mathrm{WW} \rightarrow 2 \ell 2 v$ channel [14] is sensitive from around $120 \mathrm{GeV}$ up to $600 \mathrm{GeV}$. The signature is two isolated high $p_{\mathrm{T}}$ leptons and the presence of significant missing transverse energy (MET). The Higgs mass resolution is of the order of $20 \%$. The main backgrounds in this channel are irreducible WW production, Z+jets, WZ, ZZ and W+jets. Since the Higgs boson is a scalar and due to the V-A structure of the $\mathrm{W}$ decay, the two charged leptons tend to be aligned. This favours a small difference in azimuthal angle $\Delta \phi_{\ell \ell}$ and provides a handle to discriminate the signal from the irreducible background. Events are classified according the exclusive jet multiplicity: 0,1 and 2 . To enhance the sensitivity to a Higgs boson signal, two different analyses are performed in the 0 -jet and 1-jet categories, the first exploiting a cut-based approach and the second making use of multivariate technique. The analysis of events with 0 and 1 jet is optimized for $\mathrm{gg} \rightarrow \mathrm{H} \rightarrow \mathrm{WW}$, while the one for events with 2 jets is optimized for Vector Boson Fusion (VBF) $q q \rightarrow \mathrm{H} \rightarrow \mathrm{WW}$. The cut and count approach and multivariate (Boosted Decision Tree - BDT) analysis is performed, optimized for each Higgs boson mass point. In the cut-based approach extra requirements set to maximize the expected exclusion limit for a SM Higgs boson are placed on the transverse momenta of the leading $\left(p_{\mathrm{T}}^{\ell, \max }\right)$ and the trailing leptons $\left(p_{\mathrm{T}}^{\ell, \min }\right)$, the dilepton mass $\left(\mathrm{m}_{\ell \ell}\right)$, the transverse Higgs boson mass $\left(\mathrm{m}_{\mathrm{T}}\left(\ell \ell E_{T}^{\text {miss }}\right)\right)$ and $\Delta \phi_{\ell \ell}$. In the multivariate approach a boosted decision tree (BDT) is trained for each Higgs boson mass hypothesis and jet category. In addition to the $\mathrm{W}^{+} \mathrm{W}^{-}$selection requirements, a loose selection on the dilepton mass and on the Higgs transverse mass is applied to enhance the signal-to-background ratio. The multivariate technique uses the following additional variables compared to the cut-based analysis: distance between the 2 leptons in $\eta-\phi$ space, the transverse mass of both lepton- $\mathrm{E}_{\mathrm{T}}^{\text {miss }}$ pairs; and the lepton flavors. The BDT training is performed using $\mathrm{H} \rightarrow \mathrm{W}^{+} \mathrm{W}^{-}$as signal and $\mathrm{W}^{+} \mathrm{W}^{-}$continuum as background. The main backgrounds $\left(\mathrm{W}^{+} \mathrm{W}^{-}\right.$, top, Drell Yan, $\mathrm{W}+$ jets $)$ are estimated with data-driven techniques. The uncertainty on the background normalization represent the largest source of systematics of the analysis, together with the theoretical uncertainties on the Higgs boson cross section. No evidence of Higgs boson is found and the results are interpreted as an exclusion of a wide $\mathrm{H}$ mass range. With a multivariate shape analysis, the expected exclusion range at 95\% CL is between 127 and $270 \mathrm{GeV}$ for a Standard Model Higgs boson, while the observed one is [129-270] GeV at 95\% CL using the CLs approach which has been shown in Figure 6. With the cut-based analysis, the expected exclusion range at $95 \% \mathrm{CL}$ is between 129 and $236 \mathrm{GeV}$ for a Standard Model Higgs boson, while the observed one is [132-238] GeV at 95\% CL using the CLs approach. The excess of events observed for hypothesised Higgs boson masses at the low end of the explored range makes the observed limits weaker than the expected ones.

\subsection{Combined Search}

The results of all the analyses for Higgs boson search have been combined with the frequentist CLs approach with profile likelihood test-statistics and log-normal treatment of nuisance parameters. Combined results are reported from searches for the standard model Higgs boson [18] in proton-proton collisions at $\sqrt{s}=7 \mathrm{TeV}$ in five Higgs boson decay modes: $\gamma \gamma, b \bar{b}, \tau \tau, \mathrm{WW}$, and ZZ shown in Figure 7 . The explored Higgs boson mass range is $110-600 \mathrm{GeV}$. The analysed data correspond to an integrated luminosity of $4.6-4.8 \mathrm{fb}^{-1}$. The expected excluded mass range in the absence of the standard model Higgs boson is $114.5-543 \mathrm{GeV}$ at $95 \% \mathrm{CL}$. The observed results exclude the standard model Higgs boson in the mass range $127.5-600 \mathrm{GeV}$ at $95 \% \mathrm{CL}$, and in the mass range $129-525 \mathrm{GeV}$ at $99 \% \mathrm{CL}$. An excess of events above the expected standard model background is observed at the low end of the explored mass range making the observed limits weaker than expected in the absence of a signal. The 

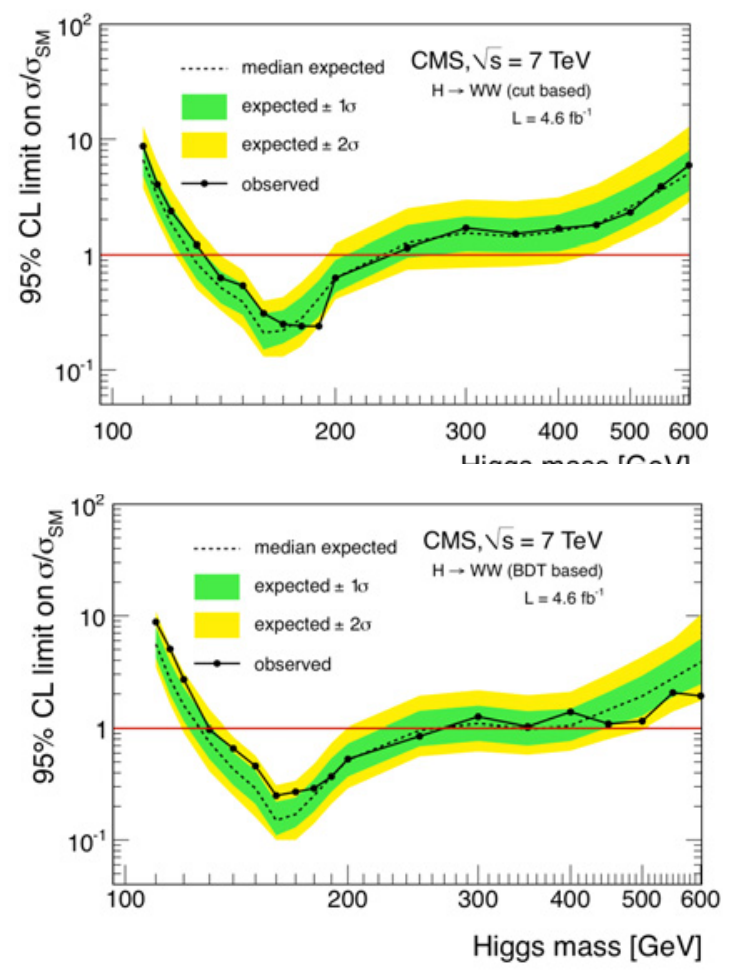

Figure 6. $95 \%$ expected and observed $\mathrm{CL}$ upper limits on the cross-section times branching ratio, $\sigma_{H} \times \mathrm{BR}\left(\mathrm{H} \rightarrow \mathrm{W}^{+} \mathrm{W}^{-} \rightarrow 2 \ell v\right)$, relative to the $\mathrm{SM}$ value using cut-based (on the left) and multivariate (on the right) event selection computed using the CLs approach.

largest excess, with a local significance of $2.8 \sigma$, is observed for a Higgs boson mass hypothesis of $125 \mathrm{GeV}$. The global significance of observing an excess with a local significance greater than $2.8 \sigma$ anywhere in the search range $110-600(110-145) \mathrm{GeV}$ is estimated to be $0.8 \sigma(2.1 \sigma)$. More data is required to ascertain the origin of this excess.

\section{Beyond SM Higgs}

A search for Higgs bosons in the minimal supersymmetric extension of the Standard Model (MSSM) has been presented for neutral and charged Higgs bosons. The decay mode considered for the search of neutral MSSM Higgs bosons [9] is to a pair of tau leptons with subsequent decays to the fully leptonic and semi-leptonic final states. For the charged Higgs boson search [19], the considered mass range is less than the top quark mass which is the search for light charged Higgs boson where the dominant decay mode is $\mathrm{H}^{ \pm} \rightarrow \tau v$ with the assumption that the decay to $\tau v$ has $100 \%$ branching ratio which is valid at high $\tan \beta$ and has $100 \%$ decay to $c \bar{s}$ at low $\tan \beta$.

\subsection{Neutral MSSM Higgs}

The tau-pair decays of the neutral Higgs bosons, having a branching fraction of roughly $10 \%$, serve as the best experimental signature for this search. The $b \bar{b}$ mode, though it has a much larger branching 

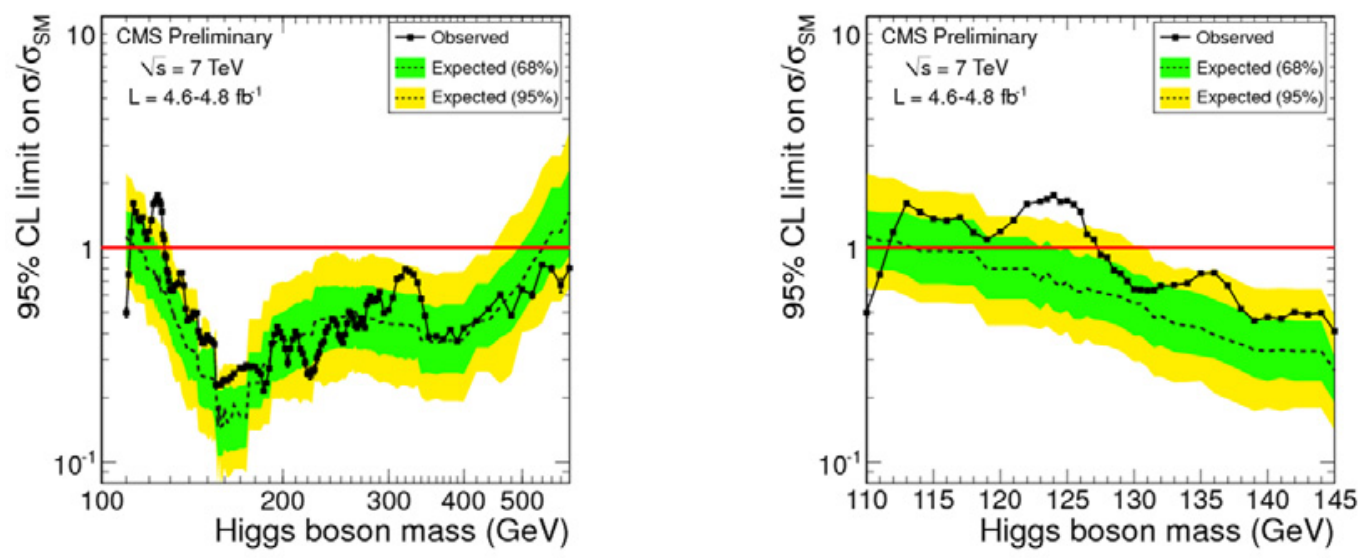

Figure 7. The $95 \% \mathrm{CL}$ upper limits on the signal strength parameter $\mu=\sigma / \sigma_{S M}$ for the SM Higgs boson hypothesis as function of the Higgs boson mass. The observed values are shown by the solid line. The dashed line indicates the expected median of results for the background only hypothesis, while the green (dark) and yellow (light) bands indicate the ranges that are expected to contain $68 \%$ and $95 \%$ of all observed excursions from the median. In the figure on the left limits are shown over the entire search range and in the figure on the right it has been zoomed to the low mass range.

fraction, suffers from an overwhelming background from multi-jet production. The analysis presented here makes use of particle flow technique which combines the information from all CMS subdetector systems to identify and reconstruct individual particles in the event, namely, muons, electrons, photons, and charged and neutral hadrons. The search is performed using the di-tau final-state signatures $\mathrm{e} \mu$, e $\tau_{h}$ and $\mu \tau_{h}$, where electrons and muons arise from leptonic $\tau$-decays and $\tau_{h}$ denotes hadronic tau lepton decays. The hadronic decay modes [8] considered for the analysis are a single charged hadron, a charged hadron in association with neutral pion via the $\rho(770)$ resonance and three charged hadrons via the $a_{1}$ resonance. The data sample for the neutral MSSM Higgs boson search has been divided into b-tag and non b-tag categories to enhance the bb $\Phi$ coupling:

- b-Tag category: At most one jet with $p_{\mathrm{T}}>30 \mathrm{GeV}$ and at least one b-tagged jet with $p_{\mathrm{T}}>20 \mathrm{GeV}$.

- Non b-Tag category: At most one jet with $p_{\mathrm{T}}>30 \mathrm{GeV}$ and no b-tagged jet with $p_{\mathrm{T}}>20 \mathrm{GeV}$.

The di-tau invariant mass spectrum [9] shows no evidence for a Higgs boson signal and hence 95\% CL upper bound on the Higgs boson production cross-section times the branching fraction to tau pairs have been set. These limits are further interpreted in the MSSM parameter space on the $\mathrm{m}_{\mathrm{A}}$-tan $\beta$ plane as shown in Figure 8 in the maximal mixing scenario of the stop-top sector with a soft SUSY breaking mass of $1 \mathrm{TeV}$ as the stop mass scale. It excludes a previously unexplored region now reaching as low as $\tan \beta=7.1$ for mass of the pseudoscalar Higgs boson $\mathrm{m}_{\mathrm{A}}=160 \mathrm{GeV}$.

\subsection{Charged MSSM Higgs}

Results are presented on the search for a light charged Higgs boson $\left(\mathrm{m}_{\mathrm{H}^{+}}<\mathrm{m}_{\text {top }}\right)$ [19] that can be produced in the decay of the top quark $\mathrm{t} \rightarrow \mathrm{H}^{+} \mathrm{b}$ and which, in turn, decays into $\tau v$ in Figure 9. The analysed data set depending on the final state correspond to an integrated luminosity up to $2.3 \mathrm{fb}^{-1}$ recorded in proton-proton collisions at $\sqrt{s}=7 \mathrm{TeV}$ by the CMS experiment at the LHC. The search is sensitive to the decays of the top quark pairs $t \bar{t} \rightarrow \mathrm{H}^{+} \mathrm{W}^{-} \mathrm{b} \overline{\mathrm{b}}$ and $\mathrm{t} \overline{\mathrm{t}} \rightarrow \mathrm{H}^{+} \mathrm{H}^{-} \mathrm{b} \overline{\mathrm{b}}$. Various final states 


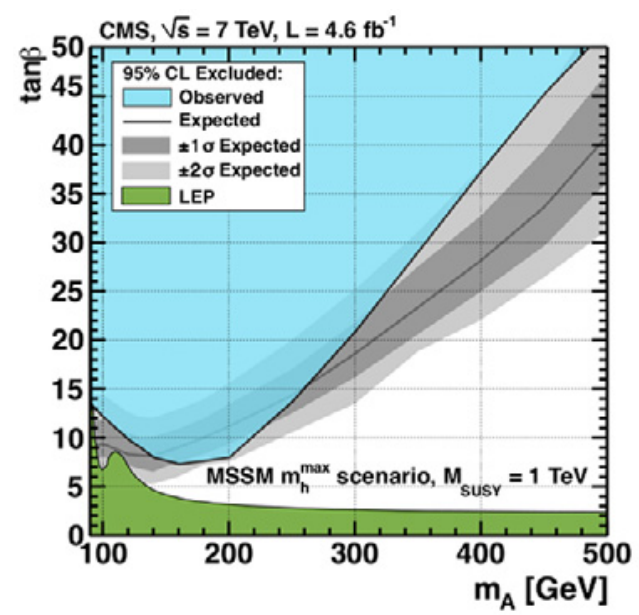

Figure 8. Region in the parameter space of $\tan \beta$ versus $\mathrm{m}_{\mathrm{A}}$ excluded at $95 \% \mathrm{CL}$ in the context of the MSSM maximal mixing scenario. The expected one- and two- standard deviation ranges and the observed $95 \% \mathrm{CL}$ upper limits are shown together with the observed excluded region.
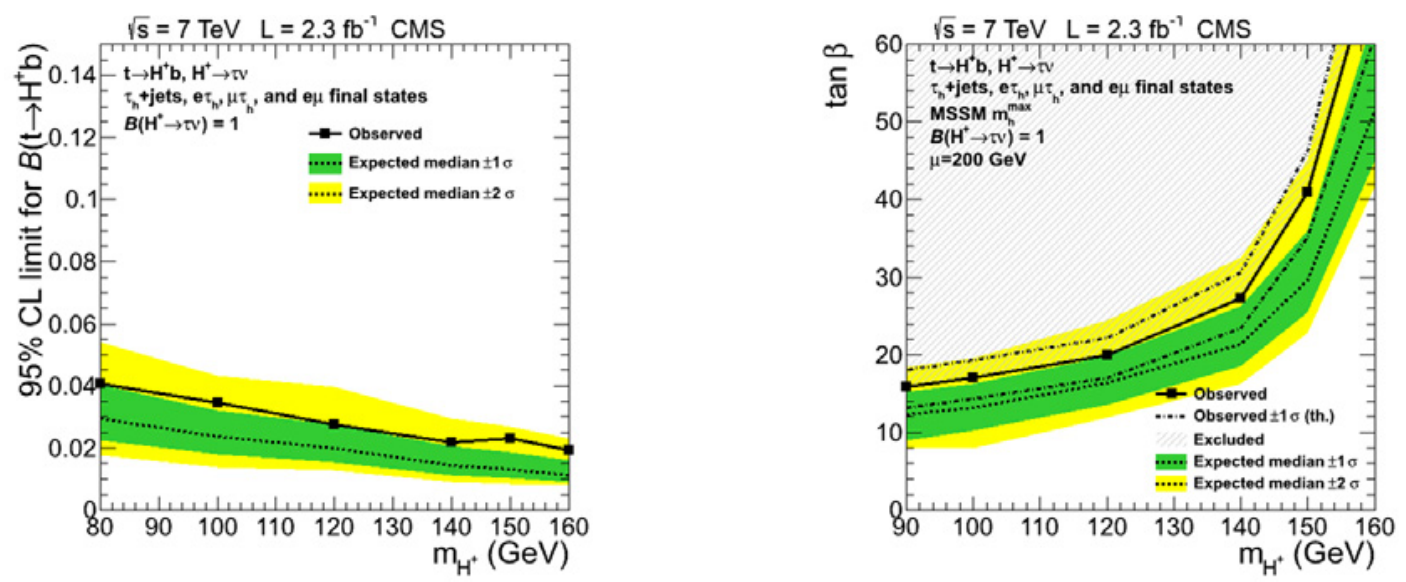

Figure 9. (Left) The upper limit on $\mathrm{BR}\left(\mathrm{t} \rightarrow \mathrm{H}^{+} \mathrm{b}\right)$ as a function of $\mathrm{m}_{\mathrm{H}^{+}}$obtained from combination of all final states. The \pm 1 and $\pm 2 \sigma$ bands around the expected limit are also shown. (Right) The exclusion region in the MSSM $\mathrm{m}_{\mathrm{H}^{+}} \tan \beta$ parameter space obtained for the MSSM $\mathrm{m}_{\mathrm{h}}^{\max }$ scenario.

have been studied separately, all requiring presence of a tau lepton from $\mathrm{H}^{+}$decays, missing transverse energy and multiple jets. The fully hadronic mode comprising hadronic tau plus jets, lepton (electron or muon $)+$ hadronic tau, and e- $\mu$ final state toplogies have been analysed and the upper limit 2-3\% on the $\mathrm{B}\left(\mathrm{t} \rightarrow \mathrm{H}^{+} \mathrm{b}\right)$ has been obtained for the charged Higgs boson mass interval $80<\mathrm{m}_{\mathrm{H}^{+}}<160 \mathrm{GeV}$ assuming $\operatorname{Br}\left(\mathrm{H}^{+} \rightarrow \tau^{+} v\right)=1$. 


\section{Conclusions}

The search for the Standard Model Higgs Boson in $7 \mathrm{TeV}$ pp collisions with the CMS detector has been presented here. The search combines the decay modes $\mathrm{H} \rightarrow \gamma \gamma, \mathrm{H} \rightarrow \tau \tau, \mathrm{H} \rightarrow b \bar{b}, \mathrm{H} \rightarrow \mathrm{WW}$ and $\mathrm{H} \rightarrow \mathrm{ZZ}$ in the mass range of 110 to $600 \mathrm{GeV}$. The SM Higgs Boson, if it exists, is limited at 95\% CL to 114.5-127.5 GeV. An excess of events has been observed at around $125 \mathrm{GeV}$ with a local significance of $2.8 \sigma$ and a significance of $2.1 \sigma$ estimated in the search range $110-145 \mathrm{GeV}$. A global significance of $0.8 \sigma$ has been estimated for the observed excess in the full search range of $110-600 \mathrm{GeV}$. The excess observed is both consistent with a background fluctuation or with the birth of a signal for Higgs Boson around $125 \mathrm{GeV}$. To ascertain the origin of this excess, more pp collision data is required.

The search for Higgs Bosons in the minimal supersymmetric extension of the Standard Model has also been presented. The search has been performed for Neutral MSSM Higgs bosons in its di-tau decay mode and low mass charged Higgs bosons in top quark decays which in turn decays to tau lepton. MSSM Higgs parameters have been significantly constrained by $\mathrm{H} \rightarrow \tau \tau$ search, now reaching as low as $\tan \beta=7.1$ for mass of the pseudoscalar Higgs boson $\mathrm{m}_{\mathrm{A}}=160 \mathrm{GeV}$. A significant constraint has also been achieved via the low mass charged Higgs boson search $\mathrm{H}^{+} \rightarrow \tau^{+} v$ on the branching fraction of the top decay to a charged Higgs boson $\mathrm{B}\left(\mathrm{t} \rightarrow \mathrm{H}^{+} \mathrm{b}\right)$ limiting it to less than 2-3\%. There has been no evidence of new physics in the beyond Standard Model Higgs sector so far.

\section{References}

[1] S. Weinberg, Phys. Rev. Lett. 19, (1967) 1264.

[2] A. Salam, p.367. Almquist and Wiksells, Stockholm, 1968.

[3] F. Englert and R. Brout, Phys. Rev. Lett. 13, (1964) 321.

[4] P. W. Higgs, Phys. Rev. Lett. 13, (1964) 508.

[5] CMS Collaboration, JINST 0803 (2008) S08004.

[6] CMS Collaboration, Phys. Lett. B 710, (2012) 403.

[7] CMS Collaboration, CMS Physics Analysis Summary CMS-PAS-HIG-12-001 (2012).

[8] CMS Collaboration, JINST 7 (2012) P01001.

[9] CMS Collaboration, Phys. Lett. B 713 (2012) 68.

[10] CMS Collaboration, JHEP 1110 (2011) 132.

[11] CMS Collaboration, Phys. Lett. B 710 (2012) 284

[12] CMS Collaboration, Phys. Rev. Lett. 108 (2012) 111804.

[13] CMS Collaboration, JHEP 03 (2012) 040.

[14] CMS Collaboration, Phys. Lett. B 710 (2012) 91.

[15] A. L. Read, CERN Yellow Report CERN-2000-005 (2000) 81.

[16] A. L. Read, J. Phys. G 28 (2002) 2693.

[17] T. Junk, Nucl. Instrum. Meth. A 434 (1999) 435, hep-ex/9902006.

[18] CMS Collaboration, CMS Physics Analysis Summary CMS-PAS-HIG-12-008 (2012).

[19] CMS Collaboration, JHEP 07 (2012) 143. 